\title{
Clinical risk factors, bacterial aetiology, and outcome of urinary tract infection in children hospitalized with diarrhoea in Bangladesh
}

\author{
R. DAS, T. AHMED, H. SAHA, L. SHAHRIN, F. AFROZE, \\ A. S. M. S. B. SHAHID, K. M. SHAHUNJA, P. K. BARDHAN AND \\ M. J. CHISTI*† \\ Nutrition and Clinical Services Division (NCSD), International Centre for Diarrhoeal Disease Research, \\ Bangladesh (icddr,b), Mohakhali, Dhaka, Bangladesh
}

Received 1 September 2016; Final revision 12 November 2016; Accepted 14 November 2016; first published online 28 December 2016

\section{SUMMARY}

Urinary tract infection (UTI) is common in children aged $<5$ years with diarrhoea, but little is known about risk factors, aetiology and outcome of such children. We aimed to evaluate these knowledge gaps of UTI in children aged $<5$ years with diarrhoea. We enrolled all children aged $<5$ years with diarrhoea admitted to Dhaka Hospital of the International Centre for Diarrhoeal Disease Research, Bangladesh, between May 2011 and April 2013, who had history of fever $\left(\geqslant 38^{\circ} \mathrm{C}\right)$ and obtained a urine sample for culture. Diarrhoea with UTI (confirmed by culture) constituted cases $(n=26)$ and those without UTI constituted controls $(n=78)$. Threefold controls were randomly selected. The case-fatality rate was comparable in cases and controls $(4 \% v s .1 \%$, $P=0 \cdot 439)$. Escherichia coli (69\%) and Klebsiella (15\%) were the most commonly isolated pathogens. Persistent diarrhoea, pneumonia and prior antibiotics use were identified as risk factors for UTI in logistic regression analysis $(P<0.05$ for all). Thus, children with diarrhoea presenting with persistent diarrhoea, pneumonia, and prior antibiotic use should be investigated for UTI for their prompt management that may reduce morbidity.

Key words: Antibiotic use prior to admission, urinary tract infection, persistent diarrhoea, pneumonia.

\section{INTRODUCTION}

Urinary tract infection (UTI) is one of the most common serious bacterial illnesses in children, especially in infants and young children [1] and is often associated with high morbidity and mortality [2]. The reported incidence of UTI is $7 \%$ in girls and $2 \%$ in

\footnotetext{
* Author for correspondence: Dr M. J. Chisti, MBBS, MMed, $\mathrm{PhD}$, Scientist \& Head, Clinical Research, Hospitals NCSD \& Clinical Lead, Intensive Care Unit \& Consultant Physician, Respiratory Ward, Dhaka Hospital, icddr,b, 68 Shaheed Tajuddin Ahmed Sarani, Mohakhali, Dhaka 1212, Bangladesh. (Email: chisti@icddrb.org)

+ Senior author.
}

boys during the first 6 years of life [3]. Studies conducted in the UK [4], Sweden [5], Finland [6], and The Netherlands [7] reported incidence rates ranging from $0 \cdot 17$ to $18 / 1000$ person-years for boys and from $0 \cdot 4$ to $66 / 1000$ person-years for girls. Due to overt clinical features, diagnosis of UTI is often delayed, which increases the risk of renal damage-related morbidity [8]. Although non-specific symptoms including vomiting, poor feeding, and diarrhoea have been postulated as signs of UTI in young children, this association has not been verified [9]. In recent studies conducted in Kingdom of Saudi Arabia and Western Iran, Escherichia coli (44.5\%), Klebsiella spp. (18.6\%), Enterobacter spp. (15\%) and Staphylococcus spp. 
(12.7\%) have been identified as the most common pathogens for UTI $[10,11]$. Of these pathogens $E$. coli is the most common responsible for UTI [12]. Studies from Ethiopia and Colombia suggest that antibiotic resistance of urinary tract pathogens is known to increase worldwide, especially to commonly used antimicrobials $[13,14]$. The increasing antibiotic resistance trends are likely to have important clinical implications for the empirical use of antibiotics [15]. Most recent studies, particularly in developing countries like Jordan and Iran, on the antimicrobial susceptibility of bacterial pathogens causing UTI in children have shown high levels of antibiotic resistance in clinical settings $[16,17]$.

Dhaka Hospital of the International Centre for Diarrhoeal Disease Research, Bangladesh (icddr,b) has cared for a number of children with diarrhoea that also have UTI but does not have any data on the burden, risk factors, bacterial aetiology and the susceptibility to antibiotics, and outcome of UTI in these children. Our aim was to evaluate the above factors in children with diarrhoea aged $<5$ years presenting with UTI.

\section{MATERIALS AND METHODS}

\section{Study design}

This was a retrospective chart review that was conducted at the Dhaka Hospital of icddr,b using the electronic database of the hospital (SHEBA). We used an unmatched case-control design and enrolled all children with diarrhoea of both sexes, aged 0-59 months, who were admitted to the intensive care unit, high-dependency unit, or longer stay ward of the hospital from May 2011 to April 2013 with history of fever $\left(\geqslant 38^{\circ} \mathrm{C}\right)$ and who had a urine culture been performed. Children with diarrhoea with UTI constituted cases and randomly selected children with diarrhoea without UTI constituted controls. Controls were randomly selected by computer randomization using SPSS v. 17.0 (SPSS Inc., Chicago) from a computerized data source of icddr,b. We used a 1:3 unmatched case-control ratio to increase the statistical power of our analyses.

\section{Study site}

The study was conducted at Dhaka Hospital of icddr, b. The description of this hospital has been given elsewhere [18].

\section{Patient management}

Children with diarrhoea and a UTI received levofloxacin/ciprofloxacin; associated other comorbidities, i.e. management of pneumonia, sepsis, severe cholera, dysentery, severe malnutrition, and other bacterial infections was performed following the hospital's guidelines $[19,20]$.

\section{Definitions}

UTI. We defined UTI in a child as the presence of significant bacteriuria [21] from a single sample of urine culture reported by the microbiology laboratory of icddr,b. Only clean-catch urine samples were collected for both cases and controls before administration of any antibiotic at hospital. Urine specimens were collected into a sterile container. Bacterial growth $<10^{5}$ c.f.u./ml from the collected urine sample were regarded as contaminants and disregarded [21].

Diarrhoea. Passage of $\geqslant 3$ abnormally loose or watery stools $/ 24 \mathrm{~h}$, and status of dehydration was defined by 'Dhaka Methods' of assessment of dehydration that is almost similar to the WHO method and approved by WHO [22].

Nosocomial infection (NI). If evidence of new infection was identified at least $48 \mathrm{~h}$ after admission, patients were categorized as NI. Evidence of new infection was categorized clinically as the development of new clinical features in hospitalized children [23, 24].

Sepsis. Presence or presumed presence of infection with hypothermia $\left(\leqslant 35.0^{\circ} \mathrm{C}\right)$ or hyperthermia $\left(\geqslant 38.5^{\circ} \mathrm{C}\right)$, tachycardia, tachypnoea, and abnormal white blood cell count (WBC) $\left(>11 \times 10^{9} / 1\right.$ or $<4 \times$ $10^{9} / 1$ or band and neutrophil ratio $\left.\geqslant 0 \cdot 10\right)[25,26]$.

Pneumonia. Initial diagnosis of pneumonia was done clinically following the WHO recommended classification of pneumonia $[20,27]$ and confirmed with radiological evidence of consolidation or patchy opacities [28] by attending hospital physicians.

Severe acute malnutrition (SAM). SAM in a child was defined if the child had severe wasting (weight for height/length $Z$ score $<-3$ of median of the WHO growth standard), or nutritional oedema.

Persistent diarrhoea (PD). PD was defined as children having acute diarrhoea lasting $\geqslant 14$ days [29]. 
Procedure. In the laboratory, each collected urine specimen was plated onto blood agar medium along with a selective and differential agar such as MacConkey agar for Gram-negative organisms [30]. Urine specimens were plated, using a $0.001 \mathrm{ml}$ calibrated loop. Plates were incubated at $35^{\circ} \mathrm{C}$ aerobically and examined at $18-24 \mathrm{~h}$, they were further incubated for another $24 \mathrm{~h}$ before a negative report was issued. Cultures were quantitated, and those microorganisms isolated in the range of $\geqslant 10^{5}$ c.f.u. $/ \mathrm{ml}$ were identified. 'Significant' bacteriuria is defined as a clean-catch, midstream specimen containing a bacterial count of $\geqslant 10^{5}$ c.f.u./ml. Bacterial identifications were performed with the API 20E system (Analytab Products, USA) [31]. The agar diffusion technique was used for antibiotic susceptibility testing. Five colonies of the test organisms were streaked on agar plates using a sterile inoculating wire loop. The appropriate multidisc, depending on whether the test organism plated will be Gram negative or Gram positive, was then placed firmly onto the surface of the dried plates, using sterile forceps. The plates were left at room temperature for $1 \mathrm{~h}$ to allow diffusion of the different antibiotics from the disk into the medium. The plates were further incubated at $37^{\circ} \mathrm{C}$ for $18-24 \mathrm{~h}$. Susceptibility pattern was interpreted using the zone sizes according to Clinical and Laboratory Standards Institute (CLSI) guidelines [32].

The identification of blood isolates was performed following standard microbiological laboratory procedure in the Dhaka Hospital, icddr,b [33].

\section{Analysis}

Case report forms were developed, pre-tested, and finalized for data acquisition. Characteristics analysed included socio-demographics (age, gender, height and weight, low socioeconomic status, source of drinking water, formula feeding, vaccination status), history of vomiting and pre-admission antibiotic use, duration of fever, clinical signs, infection indices including: WBC and immature polymorph (B), renal function by serum creatinine level, isolated uropathogens and their sensitivity, microscopic urine analysis, clinical diagnoses (sepsis, pneumonia, SAM, PD, NI) and outcome.

All data were entered into SPSS for Windows v. 17.0 and Epi-Info v. 6.0 (USD Inc., USA). Differences in proportions were compared by $\chi^{2}$ test. In normally distributed data differences of means were compared by Student's $t$ test and Mann-Whitney test was used for comparison of data that were not normally distributed. A probability of $<0.05$ was considered statistically significant. Strength of association was determined by a calculating odds ratio (OR) and $95 \%$ confidence intervals (CI). We have these statistics both in our univariate analyses and logistic regression. Initially, we performed univariate analyses of the relevant characteristics (Table 1) to identify factors that were significantly associated with UTI and finally, we performed logistic regression analysis to identify the independently associated factors of UTI in children aged $<5$ years with diarrhoea. In the logistic regression model UTI was the dependent variable and the characteristics that were significantly associated with UTI in the univariate model were considered as independent variables.

\section{Ethical standards}

Our research did not involve any interviews with patients or caregivers and it was solely a chart analysis. The data were anonymized before being received by us.

\section{RESULTS}

A total of 365 children were enrolled with study criteria. There were 26 cases and 78 controls. Thus, the prevalence of culture-proven UTI in diarrhoeal children who presented with history of fever was $7 \%$ (26/365). In all the bacterial isolates of UTI in our study, $18(69 \%)$ were E. coli, four $(15 \%)$ were Klebsiella, one each was Acinetobacter, Enterococcus, and group B streptococcus. The sensitivity of E. coli was $100 \%$ for meropenem, $93 \%$ amikacin, $79 \%$ ceftazidime, $57 \%$ levofloxacin, $20 \%$ ceftriaxone and cotrimoxazole, and $0 \%$ for ampicillin.

Cases more often received antibiotics prior to admission, presented with younger age, and pneumonia, and less often presented with documented fever on admission compared to controls (Table 1). In logistic regression analysis after adjusting for potential confounders such as younger age (6 months), severe acute malnutrition, and NI, cases more often had PD, pneumonia and received antibiotics prior to admission (Table 2). Death was comparable in cases and controls (Table 1). Other variables were also comparable in the groups (Table 1).

\section{DISCUSSION}

This study, although limited by small sample size, was able to describe our experience with the patients 
Table 1. Characteristics of cases and controls admitted to Dhaka Hospital, icddr,b.

\begin{tabular}{|c|c|c|c|c|}
\hline Characteristics & Cases $(n=26)$ & Controls $(n=78)$ & OR $(95 \% \mathrm{CI})$ (unadjusted) & $P$ value \\
\hline Male sex & $13(50)$ & $48(61 \cdot 5)$ & $0 \cdot 63(0 \cdot 23-1 \cdot 67)$ & $0 \cdot 421$ \\
\hline Age, months (median, IQR) & $6 \cdot 0(4 \cdot 8-9 \cdot 3)$ & $9 \cdot 0(4 \cdot 0-18 \cdot 0)$ & - & $0 \cdot 041$ \\
\hline Poor socioeconomic status & $17(65)$ & $57(75)$ & $0 \cdot 63(0 \cdot 22-1 \cdot 83)$ & $0 \cdot 488$ \\
\hline Source of drinking water (tube well) & $2(8)$ & $26(33)$ & $0 \cdot 27(0 \cdot 04-1 \cdot 44)$ & $0 \cdot 117$ \\
\hline Formula feeding & $3(12)$ & $7(9)$ & $2 \cdot 14(0 \cdot 28-16 \cdot 34)$ & $0 \cdot 394$ \\
\hline Vaccination & $13(50)$ & $63(81)$ & $0.36(0.08-1 \cdot 74)$ & $0 \cdot 214$ \\
\hline Documented fever on admission $\left(\geqslant 38^{\circ} \mathrm{C}\right)$ & $15(58)$ & $62(80)$ & $0 \cdot 35(0 \cdot 12-1 \cdot 01)$ & $0 \cdot 053$ \\
\hline Vomiting & $18(69)$ & $54(69)$ & $1 \cdot 00(0 \cdot 35-2 \cdot 92)$ & $0 \cdot 806$ \\
\hline Dehydrating diarrhoea & $19(73)$ & $42(54)$ & $2 \cdot 36(0 \cdot 80-6 \cdot 93)$ & $0 \cdot 135$ \\
\hline Antibiotic use prior to admission & $7(27)$ & $8(10)$ & $3 \cdot 22(0 \cdot 91-11 \cdot 51)$ & $0 \cdot 052$ \\
\hline Persistent diarrhoea & $10(39)$ & $16(21)$ & $2 \cdot 42(0 \cdot 83-7 \cdot 05)$ & $0 \cdot 067$ \\
\hline Pneumonia & $10(39)$ & $14(18)$ & $2 \cdot 86(1 \cdot 00-8 \cdot 50)$ & $0 \cdot 032$ \\
\hline SAM & $18(69)$ & $38(49)$ & $2 \cdot 37(0 \cdot 84-6 \cdot 79)$ & $0 \cdot 069$ \\
\hline Sepsis & $5(19)$ & $11(14)$ & $1 \cdot 45(0 \cdot 39-5 \cdot 25)$ & 0.539 \\
\hline Nosocomial infection & $10(39)$ & $21(27)$ & $1 \cdot 70(0 \cdot 60-4 \cdot 76)$ & $0 \cdot 386$ \\
\hline White blood cell count $\left(\mathrm{no} . / \mathrm{mm}^{3}\right)($ mean \pm s.D. $)$ & $\begin{array}{c}14373 \cdot 1 \pm \\
5652 \cdot 9\end{array}$ & $14131 \cdot 3 \pm 6736 \cdot 1$ & - & $0 \cdot 858$ \\
\hline Immature polymorph $\left(\right.$ no. $\left./ \mathrm{mm}^{3}\right)($ mean \pm s.D. $)$ & $0 \cdot 3 \pm 1 \cdot 2$ & $1 \cdot 3 \pm 4$ & - & 0.433 \\
\hline Serum creatinine level $(\mu \mathrm{mol} / \mathrm{l})$ (median, IQR) & $26 \cdot 6(21 \cdot 8-31 \cdot 7)$ & $26 \cdot 6(18 \cdot 5-43 \cdot 5)$ & - & 0.962 \\
\hline WBC in urine microscopy, per HPF (median, IQR) & $5 \cdot 5(2 \cdot 0-10 \cdot 5)$ & $8 \cdot 0(4 \cdot 0-20 \cdot 0)$ & - & $0 \cdot 072$ \\
\hline Outcome (died) & $1(4)$ & $1(1)$ & $3 \cdot 08(0 \cdot 0-117 \cdot 91)$ & 0.439 \\
\hline
\end{tabular}

OR, odds ratio; CI, confidence interval; IQR, interquartile range; SAM, severe acute malnutrition; s.D., standard deviation; HPF, high-power field.

Values given are $n(\%)$ unless indicated otherwise.

Table 2. Results of logistic regression in exploring the independent predictors of UTI in children aged $<5$ years with diarrhoea

\begin{tabular}{llll}
\hline \hline Characteristics & aOR & $95 \%$ CI & $P$ value \\
\hline Age (6 months) & $1 \cdot 043$ & $0 \cdot 980-1 \cdot 110$ & $0 \cdot 181$ \\
Antibiotic use prior to admission & $4 \cdot 208$ & $1 \cdot 216-14 \cdot 556$ & $0 \cdot 023$ \\
Severe acute malnutrition & $1 \cdot 644$ & $0 \cdot 584-4 \cdot 629$ & $0 \cdot 347$ \\
Persistent diarrhoea & $3 \cdot 364$ & $1 \cdot 163-9 \cdot 732$ & $0 \cdot 025$ \\
Pneumonia & $3 \cdot 302$ & $1 \cdot 148-9 \cdot 497$ & $0 \cdot 027$ \\
Nosocomial infection & $1 \cdot 023$ & $0 \cdot 336-3 \cdot 114$ & $0 \cdot 968$ \\
\hline \hline
\end{tabular}

aOR, Adjusted odds ratio; $\mathrm{CI}$, confidence interval

admitted for treatment of diarrhoea and UTI. We are able to identify different risk factors of UTI in children aged 0-59 months with diarrhoea. The observation of this study indicates that UTI is common in hospitalized children with diarrhoea tested for UTI. Their median age was 6 months which is consistent to our earlier observation in children without diarrhoea [34-37].

As expected, E. coli was the most common uropathogen in our study, as reported previously in a number of studies $[10,16,20,34]$. One of the important observations of this study is the association of UTI with PD. Most of the cases of PD are associated with non-gastrointestinal infections particularly UTI and acute respiratory tract infection (ARI). Those may be missed on clinical examination unless efforts are made to investigate these children [38, 39]. However, frequent association of PD has been observed with UTI or ARI or both [40]. Although we failed to identify any stool pathogen in PD cases, a previous study from Bangladesh revealed that the most common pathogen causing PD was E. coli [38]. In our study, PD developed during hospitalization and stool culture of all PD patients was performed after the 
development of PD. Initiation of antibiotics in all patients with UTI and PD might have an impact on no growth in stool culture. As these patients simultaneously presented with UTI and acute watery diarrhoea (and developed PD during hospitalization), we do not know whether diarrhoea could either be the cause or the consequence of UTI. However, a prospective study with large sample may answer this question.

Our observation that children with UTI were more likely to have received antibiotics prior to hospital admission compared to those without UTI is also consistent with other studies [41, 42]. The use of antibiotics pre-admission might have an impact for development of increased resistance bacteria [41] causing UTI or diarrhoea in our study children. However, we do not have any data that suggest the administration of antibiotics prior to hospital admission in children with diarrhoea and UTI is directly related to UTI, or that UTI is a consequence of the prior antibiotic treatment.

We observed that a higher proportion of children with diarrhoea $(39 \%)$ and UTI were associated with pneumonia than those without UTI. In our study children, both UTI and pneumonia were present simultaneously during hospitalization. We can speculate that it might be due to translocation of bacteria such as $E$. coli through transcellular and paracellular pathways of vulnerable gut in diarrhoeal children, and this might occur prior to hospitalization. This finding of our study is consistent with an earlier observation [43].

UTI in febrile children has been widely studied in different parts of the world $[13,14,21]$. An $11 \%$ prevalence in febrile children aged $<5$ years was reported by researchers in Nigeria [21]. In our study, children with diarrhoea and UTI presented less often with documented fever compared to those without UTI as most of them received antibiotics prior to hospital admission, which is consistent with previous observations [9].

Our observation of a lack of association of low socioeconomic status, source of drinking water (tube well), formula feeding, vomiting, dehydrating diarrhoea, sepsis, NI during hospitalization with UTI in diarrhoeal patients might be due to small sample size.

Our study has some limitations, including the method of urine collection. We used clean-catch urine, rather than supra-pubic aspiration, which, especially in young children, is more likely to avoid contamination of urine with stool. Second, the retrospective design of the study potentially created a selection bias as we relied on records for enrolment criteria. Third, the same design limited the sample size and probably prevented identification of subtle differences between groups for further relevant clinical risk factors of UTI in such children. Fourth, the results could only be applicable in children with diarrhoea presenting with history of fever, but could not be generalized in all diarrhoeal children.

In conclusion, our data showed that the prevalence of UTI in children aged $<5$ years with diarrhoea was $7 \%$. PD, pneumonia and history of receiving antibiotics prior to admission were independently associated with UTI in children aged $<5$ years with diarrhoea. Commonly isolated uropathogens found were E. coli and Klebsiella leading to UTI. Thus, identification of these simple clinical predictors of UTI may help clinicians for early diagnosis and prompt treatment with antibiotics in order to reduce morbidity in such populations.

\section{ACKNOWLEDGEMENTS}

This work was supported by icddr,b and its donors, which provide unrestricted support to the institution for its operations and research. Current donors providing unrestricted support include: Government of the People's Republic of Bangladesh; Canadian International Development Agency (CIDA), Swedish International Development Cooperation Agency (Sida), and the Department for International Development, UK (DFID).

We gratefully acknowledge the donors for their support and commitment to icddr,b's research efforts. We express our sincere thanks to all physicians, clinical fellows, nurses, members of the feeding team, and cleaners of the hospital for their invaluable support. We also gratefully acknowledge icddr,b library and SHEBA personnel for their tireless support and help.

\section{DECLARATION OF INTEREST}

None.

\section{REFERENCES}

1. Tseng MH, et al. Changing trend in antimicrobial resistance of pediatric uropathogens in Taiwan. Pediatrics International 2008; 50: 797-800.

2. Salmani H, et al. Pathotypic and phylogenetic study of diarrheagenic Escherichia coli and uropathogenic E. coli using multiplex polymerase chain reaction. Jundishapur Journal of Microbiology 2016; 9: e28331.

3. Craig JC, et al. Antibiotic prophylaxis and recurrent urinary tract infection in children. New England Journal of Medicine 2009; 361: 1748-1759. 
4. Stansfeld J. Clinical observations relating to incidence and aetiology of urinary-tract infections in children. British Medical Journal 1966; 1: 631.

5. Mårild S, Jodal U. Incidence rate of first-time symptomatic urinary tract infection in children under 6 years of age. Acta Paediatrica 1998; 87: 549-552.

6. Uhari M, Nuutinen M. Epidemiology of symptomatic infections of the urinary tract in children. British Medical Journal 1988; 297: 450-452.

7. Kwok WY, et al. Incidence rates and management of urinary tract infections among children in Dutch general practice: results from a nation-wide registration study. BMC Pediatrics 2006; 6: 10.

8. Neumann CG, Pryles CV. Pyelonephritis in infants and children. Autopsy experience at the Boston City Hospital, 1933-1960. American Journal of Diseases of Children 1962; 104: 215-229.

9. Shaw KN, et al. Prevalence of urinary tract infection in febrile young children in the emergency department. Pediatrics 1998; 102: e16.

10. Garout WA, et al. Urinary tract infection in children younger than 5 years. Etiology and associated urological anomalies. Saudi Medical Journal 2015; 36: 497-501.

11. Yasemi M, et al. Frequency of bacteria causing urinary tract infections and their antimicrobial resistance patterns among pediatric patients in Western Iran from 20072009. Journal of Biological Regulators and Homeostatic Agents 2014; 28: 443-448.

12. Pitout JDD. Extraintestinal pathogenic Escherichia coli: a combination of virulence with antibiotic resistance. Frontiers in Microbiology 2012; 3.

13. Alemu A, et al. Bacterial profile and drug susceptibility pattern of urinary tract infection in pregnant women at University of Gondar Teaching Hospital, Northwest Ethiopia. BMC Research Notes 2012; 5: 197.

14. Velez Echeverri C, et al. Resistance profile for pathogens causing urinary tract infection in a pediatric population, and antibiotic treatment response at a university hospital, 2010-2011. Colombia Medica (Cali, Colombia) 2014; 45: 39-44.

15. Farajnia $\mathbf{S}$, et al. Causative agents and antimicrobial susceptibilities of urinary tract infections in the northwest of Iran. International Journal of Infectious Diseases 2009; 13: 140-144.

16. Al-Mardeni RI, et al. Empirical treatment for pediatric urinary tract infection and resistance patterns of uropathogens, in Queen Alia hospital and Prince A'Isha military center-Jordan. Saudi Journal of Kidney Diseases and Transplantation 2009; 20: 135-139.

17. Al-Mendalawi MD. Antibiotic resistance pattern and empirical therapy for urinary tract infections in children. Saudi Medical Journal 2008; 29: 1520.

18. Chisti MJ, et al. A prospective study of the prevalence of tuberculosis and bacteraemia in Bangladeshi children with severe malnutrition and pneumonia including an evaluation of Xpert MTB/RIF Assay. PLoS ONE 2014; 9: e93776.

19. Chisti MJ, et al. Bubble continuous positive airway pressure for children with severe pneumonia and hypoxaemia in Bangladesh: an open, randomised controlled trial. Lancet 2015; 386: 1057-1065.

20. WHO. Pocket Book for Hospital Care of Children: Guidelines for the Management of Common Illness with Limited Resources. World Health Organization, Geneva, Switzerland, 2013.

21. Ibeneme CA, et al. Urinary tract infection in febrile under five children in Enugu, South Eastern Nigeria. Nigerian Journal of Clinical Practice 2014; 17: 624-628.

22. Alam NH, Ashraf H. Treatment of infectious diarrhea in children. Paediatric Drugs 2003; 5: 151-165.

23. Shahunja KM, et al. Experience with nosocomial infection in children under 5 treated in an urban diarrheal treatment center in Bangladesh. Global Pediatric Health 2016; 3: 2333794x16634267.

24. Fagon JY, et al. Nosocomial pneumonia and mortality among patients in intensive care units. Journal of American Medical Association 1996; 275: 866-869.

25. Chisti MJ, et al. Clinical predictors and outcome of hypoxaemia among under-five diarrhoeal children with or without pneumonia in an urban hospital, Dhaka, Bangladesh. Tropical Medicine \& International Health 2012; 17: 106-111.

26. Dellinger RP, et al. Surviving sepsis campaign: international guidelines for management of severe sepsis and septic shock: 2012. Critical Care Medicine 2013; 41: 580-637.

27. Wandi F, Peel D, Duke T. Hypoxaemia among children in rural hospitals in Papua New Guinea: epidemiology and resource availability - a study to support a national oxygen programme. Annals of Tropical Paediatrics 2006; 26: 277-284.

28. Chisti MJ, et al. Clinical and laboratory features of radiologic pneumonia in severely malnourished infants attending an urban diarrhea treatment center in Bangladesh. Pediatric Infectious Disease Journal 2010; 29: 174-177.

29. Anon. WHO Guidelines Approved by the Guidelines Review Committee. In: Pocket Book of Hospital Care for Children: Guidelines for the Management of Common Childhood Illnesses. Geneva: World Health Organization, 2013.

30. Fung JC, et al. Primary culture media for routine urine processing. Journal of Clinical Microbiology 1982; 16: 632-636.

31. Ahmed D, et al. Recurrent urinary tract infection due to co-infection with extended spectrum $\beta$-lactamase-producer uropathogenic Escherichia coli and enteroaggregative E. coli. JMM Case Reports 2014; 1.

32. Shaifali I, et al. Antibiotic susceptibility patterns of urinary pathogens in female outpatients. North American Journal of Medical Sciences 2012; 4: 163-169.

33. Shahunja KM, et al. Predictors of death in under-five children with sepsis attending an urban diarrheal treatment centre in Bangladesh. Food and Nutrition Sciences 2013; 04: 709-714.

34. Heffner VA, Gorelick MH. Pediatric urinary tract infection. Clinical Pediatric Emergency Medicine; 9: 233-237. 
35. Hendaus MA, et al. Risk of urinary tract infection in infants and children with acute bronchiolitis. Paediatrics \& Child Health 2015; 20: e25-29.

36. Mirsoleymani SR, et al. Bacterial pathogens and antimicrobial resistance patterns in pediatric urinary tract infections: a four-year surveillance study (2009-2012). International Journal of Pediatrics 2014; 2014: 126142.

37. Roman HK, Chang PW, Schroeder AR. Diagnosis and management of bacteremic urinary tract infection in infants. Hospital Pediatrics 2015; 5: 1-8.

38. Ashraf b H, et al. Persistent diarrhoea: associated infection and response to a low lactose diet. Journal of Tropical Pediatrics 2002; 48: 142-148.

39. Chisti MJ, et al. Predictors of bacteremia in infants with diarrhea and systemic inflammatory response syndrome attending an urban diarrheal treatment center in a developing country. Pediatric Critical Care Medicine 2010; 11: 92-97.
40. Sibal A, et al. Associated infections in persistent diarrhoea another perspective. Journal of Tropical Pediatrics 1996; 42: 64-67.

41. Arslan H, et al. Risk factors for ciprofloxacin resistance among Escherichia coli strains isolated from communityacquired urinary tract infections in Turkey. Journal of Antimicrobial Chemotherapy 2005; 56: 914-918.

42. Marcus N, et al. Community-acquired Pseudomonas aeruginosa urinary tract infections in children hospitalized in a tertiary center: relative frequency, risk factors, antimicrobial resistance and treatment. Infection 2008; 36: 421-426.

43. Sood A, et al. Incidence, admission rates, and economic burden of pediatric emergency department visits for urinary tract infection: data from the nationwide emergency department sample, 2006 to 2011. Journal of Pediatric Urology 2015; 11: 246.e1-8. 